\title{
ASYMPTOTIC RUIN PROBABILITIES FOR A BIVARIATE LÉVY-DRIVEN RISK MODEL WITH HEAVY-TAILED CLAIMS AND RISKY INVESTMENTS
}

\author{
XUEMIAO HAO, ${ }^{*}$ University of Manitoba \\ QIHE TANG,** University of Iowa
}

\begin{abstract}
Consider a general bivariate Lévy-driven risk model. The surplus process $Y$, starting with $Y_{0}=x>0$, evolves according to $\mathrm{d} Y_{t}=Y_{t-} \mathrm{d} R_{t}-\mathrm{d} P_{t}$ for $t>0$, where $P$ and $R$ are two independent Lévy processes respectively representing a loss process in a world without economic factors and a process describing the return on investments in real terms. Motivated by a conjecture of Paulsen, we study the finite-time and infinitetime ruin probabilities for the case in which the loss process $P$ has a Lévy measure of extended regular variation and the stochastic exponential of $R$ fulfills a moment condition. We obtain a simple and unified asymptotic formula as $x \rightarrow \infty$, which confirms Paulsen's conjecture.
\end{abstract}

Keywords: Asymptotics; (extended) regular variation; finite-time and infinite-time ruin probabilities; Lévy process; stochastic difference equation; tail probability

2010 Mathematics Subject Classification: Primary 91B30

Secondary 60G51; 91B28

\section{Introduction}

Consider a bivariate Lévy-driven risk model in which the surplus process, $Y$, of an insurance company is modeled by

$$
Y_{t}=x-P_{t}+\int_{0}^{t} Y_{s-} \mathrm{d} R_{s}, \quad t>0,
$$

with $Y_{0}=x>0$ the initial surplus level, and $P$ and $R$ two independent Lévy processes respectively representing a loss process in a world without economic factors and a process describing the return on investments in real terms. See Paulsen (1998a), (1998b), (2002), (2008) for detailed explanations.

This model does not mean that the surplus must be completely invested in a risky asset. An understanding of the stochastic process $R$ is as follows. Consider a financial market consisting of a risk-free bond with price $S_{0, t}$ and $d$ risky stocks with prices $S_{k, t}$ for $k=1, \ldots, d$. Denote by $\pi_{0}$ the proportion of the surplus invested in the bond and by $\pi_{k}$ the proportion invested in stock $k$. Thus, $\pi=\left(\pi_{0}, \pi_{1}, \ldots, \pi_{d}\right)$, called a relative investment portfolio and assumed to be

Received 8 February 2011; revision received 11 March 2012.

* Postal address: Asper School of Business, University of Manitoba, 181 Freedman Crescent, Winnipeg, Manitoba R3T 5V4, Canada. Email address: xuemiao.hao@ad.umanitoba.ca

** Postal address: Department of Statistics and Actuarial Science, University of Iowa, 241 Schaeffer Hall, Iowa City, IA 52242, USA. Email address: qihe-tang@uiowa.edu 
time invariant, satisfies $\pi_{0}+\pi_{1}+\cdots+\pi_{d}=1$. Then the differential in (1.1) is

$$
\mathrm{d} R_{t}=\sum_{k=0}^{d} \pi_{k} \frac{\mathrm{d} S_{k, t}}{S_{k, t-}}, \quad t>0 .
$$

In particular, consider the Black-Scholes market with

$$
\begin{aligned}
& \mathrm{d} S_{0, t}=r S_{0, t} \mathrm{~d} t, \quad t>0, \\
& \mathrm{~d} S_{k, t}=S_{k, t}\left(\mu_{k} \mathrm{~d} t+\sigma_{k} \mathrm{~d} W_{k, t}\right), \quad t>0,
\end{aligned}
$$

where $\boldsymbol{W}_{t}=\left(W_{1, t}, \ldots, W_{d, t}\right)$ is a $d$-dimensional Wiener process, $r \geq 0,-\infty<\mu_{k}<\infty$, and $\sigma_{k}>0$ for $k=1, \ldots, d$. Then

$$
\mathrm{d} R_{t}=\pi_{0} r \mathrm{~d} t+\sum_{k=1}^{d} \pi_{k}\left(\mu_{k} \mathrm{~d} t+\sigma_{k} \mathrm{~d} W_{k, t}\right), \quad t>0 .
$$

Therefore, the assumption that $R$ is a Lévy process is fulfilled in this particular case.

The solution of (1.1) is given by

$$
Y_{t}=\mathrm{e}^{\tilde{R}_{t}}\left(x-\int_{0}^{t} \mathrm{e}^{-\tilde{R}_{s}} \mathrm{~d} P_{S}\right),
$$

where $\tilde{R}$, also a Lévy process, is the logarithm of the stochastic exponential (also called the Doléans-Dade exponential) of $R$; see, e.g. Protter (2005) for details. For simplicity, we write

$$
Z_{t}=\int_{0}^{t} \mathrm{e}^{-\tilde{R}_{s}} \mathrm{~d} P_{s}
$$

so that $Y_{t}=\mathrm{e}^{\tilde{R}_{t}}\left(x-Z_{t}\right)$. The stochastic process $Z$ is usually called the discounted net loss process. We will start with (1.2) instead of (1.1), as has been done by many researchers, including Kalashnikov and Norberg (2002) and Klüppelberg and Kostadinova (2008).

We are interested in the asymptotic behavior of the finite-time and infinite-time ruin probabilities of this bivariate Lévy-driven risk model. As usual, the finite-time ruin probability is defined as

$$
\psi(x, T)=\mathrm{P}\left(\inf _{0 \leq t \leq T} Y_{t}<0 \mid Y_{0}=x\right), \quad T \geq 0,
$$

and the infinite-time ruin probability is defined as

$$
\psi(x, \infty)=\lim _{T \rightarrow \infty} \psi(x, T)=\mathrm{P}\left(\inf _{0 \leq t<\infty} Y_{t}<0 \mid Y_{0}=x\right)
$$

Introduce

$$
Z_{T}^{*}=\sup _{0 \leq t \leq T} Z_{t}=\sup _{0 \leq t \leq T} \int_{0}^{t} \mathrm{e}^{-\tilde{R}_{s}} \mathrm{~d} P_{s}, \quad 0 \leq T \leq \infty,
$$

where the supremum is taken over $0 \leq t<\infty$ if $T=\infty$. Noting that $Y_{t}<0$ and $Z_{t}>x$ are equivalent, we have

$$
\psi(x, T)=\mathrm{P}\left(Z_{T}^{*}>x\right), \quad 0<T \leq \infty .
$$

In this paper we aim at a simple and unified asymptotic expression for $\psi(x, T)$ with $0<T \leq \infty$ as the initial surplus level $x$ becomes large. 


\section{Main results}

Hereafter, all limit relationships are for $x \rightarrow \infty$ unless otherwise stated. For two positive functions $a(\cdot)$ and $b(\cdot)$, we write $a(x) \sim b(x)$ if $\lim a(x) / b(x)=1, a(x) \lesssim b(x)$ or $b(x) \gtrsim a(x)$ if $\limsup a(x) / b(x) \leq 1$, and $a(x) \asymp b(x)$ if $0<\liminf a(x) / b(x) \leq$ $\lim \sup a(x) / b(x)<\infty$. For a real number $x$, we write $x^{+}=x \vee 0$ and $x^{-}=(-x) \vee 0$ as the positive and negative parts of $x$, respectively.

For a general Lévy process $L$, its characteristic exponent, $\Psi_{L}(u)=-\log \left(\mathrm{E}^{\mathrm{i} u L_{1}}\right)$, has the Lévy-Khintchine representation

$$
\Psi_{L}(u)=\mathrm{i} l u+\frac{1}{2} \sigma^{2} u^{2}+\int_{-\infty}^{\infty}\left(1-\mathrm{e}^{\mathrm{i} u x}+\mathrm{i} u x 1_{(|x|<1)}\right) v(\mathrm{~d} x)
$$

with $l \in \mathbb{R}, \sigma \geq 0$, and Lévy measure $v$ on $\mathbb{R} \backslash\{0\}$ satisfying $\int_{-\infty}^{\infty}\left(x^{2} \wedge 1\right) v(\mathrm{~d} x)<\infty$. We further denote by

$$
\varphi_{L}(u)=-\Psi_{L}(\mathrm{i} u)=\log \mathrm{Ee}^{-u L_{1}}
$$

the Laplace exponent of $L$. Clearly, $\log \mathrm{Ee}^{-u L_{t}}=t \varphi_{L}(u)$ for every $t \geq 0$.

Now we turn to the loss process $P$, which we assume to be a Lévy process. When its Lévy measure satisfies $\bar{v}_{P}(1)=v_{P}((1, \infty))>0$, introduce $\Pi_{P}(\cdot)=v_{P}(\cdot) 1_{(1, \infty)} / \bar{v}_{P}(1)$, which is a proper probability measure on $(1, \infty)$. We assume that $\Pi_{P}$ is of extended regular variation (ERV). Formally, a distribution $F$ belongs to the class $\operatorname{ERV}(-\alpha,-\beta)$ for some $0 \leq \alpha \leq \beta<\infty$ if $\bar{F}(x)=1-F(x)>0$ holds for all $x$ and the relations

$$
v^{-\beta} \leq \liminf \frac{\bar{F}(v x)}{\bar{F}(x)} \leq \lim \sup \frac{\bar{F}(v x)}{\bar{F}(x)} \leq v^{-\alpha}
$$

hold for all $v \geq 1$. Note that relations (2.2) with $\alpha=\beta$ define the class $\mathcal{R}_{-\alpha}$ of distributions of regular variation.

Our main result is as follows.

Theorem 2.1. Consider the bivariate Lévy-driven risk process $Y$ given by (1.2), where $P$ and $\tilde{R}$ are two independent Lévy processes. Assume that $\Pi_{P} \in \operatorname{ERV}(-\alpha,-\beta)$ for some $0<\alpha \leq \beta<\infty$.

(i) If $\mathrm{E}\left(\mathrm{e}^{-(\beta+\varepsilon) \tilde{R}_{1}}\right)<1$ for some $\varepsilon>0$ then it holds for every $T \in(0, \infty)$ that

$$
\psi(x, T) \sim \lambda \int_{0}^{T} \mathrm{P}\left(X \mathrm{e}^{-\tilde{R}_{t}}>x\right) \mathrm{d} t,
$$

where $\lambda=\bar{v}_{P}(1)$ and $X$ is distributed by $\Pi_{P}$, and independent of $P$ and $\tilde{R}$.

(ii) Furthermore, if $\mathrm{E}\left(\mathrm{e}^{-(\alpha-\varepsilon) \tilde{R}_{1}} \vee \mathrm{e}^{-(\beta+\varepsilon) \tilde{R}_{1}}\right)<1$ for some $\varepsilon \in(0, \alpha)$ then relation (2.3) also holds for $T=\infty$.

The condition $\Pi_{P} \in \operatorname{ERV}(-\alpha,-\beta)$ means that insurance claims are heavy tailed. Roughly speaking, the exponential moment condition in each case means that insurance claims control the uncertainty of negative returns of investments. When $\alpha=\beta$, it is easy to see that the exponential moment conditions in both cases are equivalent. For this case, the statement of Theorem 2.1 is simplified to the following.

Corollary 2.1. Let $\alpha=\beta$ in Theorem 2.1. Then it holds for every $T \in(0, \infty]$ that

$$
\psi(x, T) \sim \frac{1-\mathrm{e}^{\varphi_{\tilde{R}}(\alpha) T}}{-\varphi_{\tilde{R}}(\alpha)} \bar{v}_{P}(x) .
$$


Relation (2.4) with $T=\infty$ was conjectured by Paulsen (2002, Theorem 3.2(b) and Remark 3.2(b)). Therefore, our work shows that Paulsen's conjecture is indeed true.

Admittedly, the class ERV is marginally larger than the class $\mathcal{R}$, but it incurs a lot more technicalities to the study. A self-contained proof targeting Corollary 2.1 only can be much simpler than the proof of Theorem 2.1 given below. However, we have reasons to believe that the ruin probabilities for the more general subexponential case will asymptotically behave in the form of (2.3), rather than (2.4). Therefore, we carry out our research within the class ERV, hoping that it will offer insights into the subexponential case.

The asymptotic behavior of the ruin probabilities in the presence of investments has been extensively investigated in the literature. Early works focused on a special case in which $P$ is a compound Poisson process with subexponential jumps and $R$ is a deterministic linear function (corresponding to a constant rate of compound interest); see Klüppelberg and Stadtmüller (1998), Asmussen (1998), Kalashnikov and Konstantinides (2000), and Konstantinides et al. (2002). Later on, for the subexponential case, Tang (2005) established a formula for the finitetime ruin probability, which is in line with the formula for the infinite-time ruin probability. The formulae obtained in these papers are essentially the same as (2.3) with $\tilde{R}$ replaced by a deterministic linear function.

It is more interesting to consider that the insurance company makes both risk-free and risky investments. For a very general case, Kalashnikov and Norberg (2002) proved that the infinitetime ruin probability necessarily decays to 0 as a power function as the initial surplus level increases no matter what tail behavior the jumps of $P$ have. Frolova et al. (2002) obtained an explicit asymptotic formula for the infinite-time ruin probability for the case in which $P$ is a compound Poisson process with exponential (hence light-tailed) jumps and $R$ is a Wiener process with drift.

Most of the recent research focuses on the case in which $P$ has regularly varying jumps. For example, Paulsen (2002) showed relation (2.4) with $T=\infty$ for a special case in which $P$ is a compound Poisson process with regularly varying jumps and $R$ (or, equivalently, $\tilde{R}$ ) is a Wiener process with drift. Klüppelberg and Kostadinova (2008) extended this result to a general Lévy process $\tilde{R}$. Heyde and Wang (2009) obtained similar results to our relations (2.3) and (2.4) with $T<\infty$ for the case in which $P$ is a compound Poisson process with heavy-tailed jumps and $\tilde{R}$ is a general Lévy process. Tang et al. (2010) established a result similar to our relation (2.3) for both $T<\infty$ and $T=\infty$ for the case in which $P$ is a compound renewal process with regularly varying jumps and $\tilde{R}$ is a general Lévy process. Albrecher et al. (2012) also investigated the asymptotic behavior of the infinite-time ruin probability and related quantities for the case in which $P$ is a compound renewal process having light-tailed or heavy-tailed jumps and $\tilde{R}$ is a Brownian motion with drift. Hult and Lindskog (2011) considered a more general case in which $P$ is a Lévy process with regularly varying jumps and $R$ is a semimartingale, and they established an asymptotic formula for the finite-time ruin probability, which holds uniformly for all $R$ with the stochastic exponential $\mathrm{e}^{\tilde{R}_{t}}$ fulfilling a certain moment condition. See also Asmussen and Albrecher (2010, Sections VIII.5-6) for a brief review of ruin theory in the presence of investments.

We are going to prepare some lemmas in Section 3, and then prove Theorem 2.1 and Corollary 2.1 in Section 4.

\section{Lemmas}

In this section we prepare some lemmas for the main result. The first lemma below describes some well-known properties of distributions of extended regular variation; see Bingham et al. (1987, Proposition 2.2.3) and Tang and Tsitsiashvili (2003, Lemma 3.5). 
Lemma 3.1. Suppose that $F \in \operatorname{ERV}(-\alpha,-\beta)$ for some $0<\alpha \leq \beta<\infty$.

(i) For every $\varepsilon \in(0, \alpha)$ and every $b>1$, there exists some $x_{0}>0$ such that the inequalities

$$
\frac{1}{b}\left(y^{-(\alpha-\varepsilon)} \wedge y^{-(\beta+\varepsilon)}\right) \leq \frac{\bar{F}(x y)}{\bar{F}(x)} \leq b\left(y^{-(\alpha-\varepsilon)} \vee y^{-(\beta+\varepsilon)}\right)
$$

hold whenever $x>x_{0}$ and $x y>x_{0}$.

(ii) It holds for every $\varepsilon>0$ that $\bar{F}(x)=o\left(x^{-(\alpha-\varepsilon)}\right)$ and $x^{-(\beta+\varepsilon)}=o(\bar{F}(x))$.

A feature of the following lemma is that inequality (3.1) holds uniformly for all nonnegative random variables $Y$ independent of $X$.

Lemma 3.2. Let $X$ be a real-valued random variable whose distribution belongs to the class $\operatorname{ERV}(-\alpha,-\beta)$ for some $0<\alpha \leq \beta<\infty$. Then, for every $\varepsilon \in(0, \alpha)$ and every $b>1$, there exists some $x_{0}=x_{0}(\varepsilon, b)>0$ such that, for all $x>x_{0}$ and all nonnegative random variables $Y$ independent of $X$,

$$
\frac{\mathrm{P}(X Y>x)}{\mathrm{P}(X>x)} \leq b \mathrm{E}\left(Y^{\alpha-\varepsilon} \vee Y^{\beta+\varepsilon}\right) .
$$

Proof. For arbitrarily chosen $b^{\prime} \in(1, b)$, by Lemma 3.1(i), there exists some $x_{0}^{\prime}>0$ such that, for all $x>x_{0}^{\prime}$,

$$
\begin{aligned}
\mathrm{P}(X Y>x) & \leq \mathrm{P}\left(X Y>x, Y \leq \frac{x}{x_{0}^{\prime}}\right)+\mathrm{P}\left(Y>\frac{x}{x_{0}^{\prime}}\right) \\
& \leq b^{\prime} \mathrm{P}(X>x) \mathrm{E}\left(Y^{\alpha-\varepsilon} \vee Y^{\beta+\varepsilon}\right)+\left(\frac{x}{x_{0}^{\prime}}\right)^{-(\beta+\varepsilon)} \mathrm{E} Y^{\beta+\varepsilon} .
\end{aligned}
$$

Then inequality (3.1) follows since $x^{-(\beta+\varepsilon)}=o(\mathrm{P}(X>x))$ by Lemma 3.1(ii).

By following similar lines to the proof of Lemma 4.4.2 of Samorodnitsky and Taqqu (1994), we obtain the following result.

Lemma 3.3. Let $(X, Y)$ be jointly distributed random variables. If the distribution of $X$ belongs to the class $\operatorname{ERV}(-\alpha,-\beta)$ for some $0 \leq \alpha \leq \beta<\infty$ and $\mathrm{P}(|Y|>x)=o(\mathrm{P}(X>x))$, then $\mathrm{P}(X+Y>x) \sim \mathrm{P}(X>x)$.

The tail behavior of randomly weighted sums of heavy-tailed random variables has become a hot topic in applied probability since Resnick and Willekens (1991). A very recent work on the topic is Olvera-Cravioto (2012). The next lemma summarizes several known results.

Lemma 3.4. Let $\left\{X_{k}, k \in \mathbb{N}\right\}$ be a sequence of independent and identically distributed (i.i.d.) random variables with common distribution $F$, and let $\left\{\omega_{k}, k \in \mathbb{N}\right\}$ be another sequence of nonnegative random variables, nondegenerate at zero and independent of $\left\{X_{k}, k \in \mathbb{N}\right\}$. Assume that $F \in \operatorname{ERV}(-\alpha,-\beta)$ for some $0<\alpha \leq \beta<\infty$ and that one of the following two conditions holds:

(i) in the case $\beta \in(0,1)$, there exists some $\varepsilon \in(0, \alpha)$ such that

$$
\sum_{k=1}^{\infty} \mathrm{E}\left(\omega_{k}^{\alpha-\varepsilon} \vee \omega_{k}^{\beta+\varepsilon}\right)<\infty
$$


(ii) in the case $\beta \in[1, \infty)$, there exists some $\varepsilon \in(0, \alpha)$ such that

$$
\sum_{k=1}^{\infty}\left(\mathrm{E}\left(\omega_{k}^{\alpha-\varepsilon} \vee \omega_{k}^{\beta+\varepsilon}\right)\right)^{1 /(\beta+\varepsilon)}<\infty
$$

Then

$$
\mathrm{P}\left(\max _{1 \leq n<\infty} \sum_{k=1}^{n} \omega_{k} X_{k}>x\right) \sim \mathrm{P}\left(\sum_{k=1}^{\infty} \omega_{k} X_{k}^{+}>x\right) \sim \sum_{k=1}^{\infty} \mathrm{P}\left(\omega_{k} X_{k}>x\right) .
$$

Furthermore, the distributions of $\max _{1 \leq n<\infty} \sum_{k=1}^{n} \omega_{k} X_{k}$ and $\sum_{k=1}^{\infty} \omega_{k} X_{k}^{+}$both belong to the class $\operatorname{ERV}(-\alpha,-\beta)$.

Proof. The second relation in (3.2) has been proved in Theorem 3.1(b) of Zhang et al. (2009).

Let us check the extended regular variation of the distribution, denoted by $F_{\omega}^{+}$, of $\sum_{k=1}^{\infty} \omega_{k} X_{k}^{+}$. It is easy to prove that, for each $k \in \mathbb{N}$, the relation $\mathrm{P}\left(\omega_{k} X_{k}>x\right) \asymp \bar{F}(x)$ holds and the distribution of $\omega_{k} X_{k}$ belongs to the class $\operatorname{ERV}(-\alpha,-\beta)$; see Cline and Samorodnitsky (1994, Theorem 3.5) for these facts. By Lemma 3.2, for every $b>1$, there exists some $x_{0}>0$ such that the inequality

$$
\mathrm{P}\left(\omega_{k} X_{k}>x\right) \leq b \bar{F}(x) \mathrm{E}\left(\omega_{k}^{\alpha-\varepsilon} \vee \omega_{k}^{\beta+\varepsilon}\right)
$$

holds for all $k \in \mathbb{N}$ and all $x>x_{0}$. Hence, for arbitrarily given $\delta>0$, all $x>x_{0}$, and all large $n$,

$$
\sum_{k=n}^{\infty} \mathrm{P}\left(\omega_{k} X_{k}>x\right) \leq b \bar{F}(x) \sum_{k=n}^{\infty} \mathrm{E}\left(\omega_{k}^{\alpha-\varepsilon} \vee \omega_{k}^{\beta+\varepsilon}\right) \leq \delta \mathrm{P}\left(\omega_{1} X_{1}>x\right) .
$$

By the second relation in (3.2), inequalities (3.3), and the fact that each $\omega_{k} X_{k}$ follows a distribution in $\operatorname{ERV}(-\alpha,-\beta)$, it holds for arbitrarily fixed $v>1$ and some large $n_{0}$ that

$$
\frac{\bar{F}_{\omega}^{+}(v x)}{\bar{F}_{\omega}^{+}}(x) \sim \frac{\sum_{k=1}^{\infty} \mathrm{P}\left(\omega_{k} X_{k}>v x\right)}{\sum_{k=1}^{\infty} \mathrm{P}\left(\omega_{k} X_{k}>x\right)} \lesssim(1+\delta) \frac{\sum_{k=1}^{n_{0}} \mathrm{P}\left(\omega_{k} X_{k}>v x\right)}{\sum_{k=1}^{n_{0}} \mathrm{P}\left(\omega_{k} X_{k}>x\right)} \lesssim(1+\delta) v^{-\alpha} .
$$

Symmetrically,

$$
\frac{\bar{F}_{\omega}^{+}(v x)}{\bar{F}_{\omega}^{+}}(x) \gtrsim \frac{1}{1+\delta} v^{-\beta} .
$$

The arbitrariness of $\delta$ implies that $F_{\omega}^{+} \in \operatorname{ERV}(-\alpha,-\beta)$.

The extended regular variation of the distribution of $\max _{1 \leq n<\infty} \sum_{k=1}^{n} \omega_{k} X_{k}$ easily follows from the extended regular variation of $F_{\omega}^{+}$and the first relation in (3.2).

It remains to verify the first relation in (3.2). Since the inequality

$$
\sum_{k=1}^{\infty} \omega_{k} X_{k}^{+} \geq \max _{1 \leq n<\infty} \sum_{k=1}^{n} \omega_{k} X_{k}
$$

is obvious, we only need to prove that

$$
\mathrm{P}\left(\max _{1 \leq n<\infty} \sum_{k=1}^{n} \omega_{k} X_{k}>x\right) \gtrsim \sum_{k=1}^{\infty} \mathrm{P}\left(\omega_{k} X_{k}>x\right) .
$$


Actually, by Theorem 3.2 of Chen and Yuen (2009), it holds for all $n \in \mathbb{N}$ that

$$
\mathrm{P}\left(\sum_{k=1}^{n} \omega_{k} X_{k}>x\right) \sim \sum_{k=1}^{n} \mathrm{P}\left(\omega_{k} X_{k}>x\right) .
$$

By (3.3) and (3.5), it holds for arbitrarily given $\delta>0$ and some large $n_{0}$ that

$$
\mathrm{P}\left(\max _{1 \leq n<\infty} \sum_{k=1}^{n} \omega_{k} X_{k}>x\right) \gtrsim \sum_{k=1}^{n_{0}} \mathrm{P}\left(\omega_{k} X_{k}>x\right) \gtrsim \frac{1}{1+\delta} \sum_{k=1}^{\infty} \mathrm{P}\left(\omega_{k} X_{k}>x\right) .
$$

Then the arbitrariness of $\delta$ implies (3.4).

Consider the compound Poisson process

$$
C_{t}=\sum_{k=1}^{N_{t}} X_{k}, \quad t \geq 0,
$$

where $\left\{X_{k}, k \in \mathbb{N}\right\}$ is a sequence of i.i.d. random variables with generic random variable $X$ and common distribution $F$, while $\left\{N_{t}, t \geq 0\right\}$ is a Poisson process, independent of $\left\{X_{k}, k \in \mathbb{N}\right\}$, with intensity $\lambda>0$. The following lemma plays a crucial role in proving Theorem 2.1.

Lemma 3.5. Let $C$ be a compound Poisson process given by (3.6), and let $L$ be a Lévy process independent of $C$. If $F \in \operatorname{ERV}(-\alpha,-\beta)$ for some $0<\alpha \leq \beta<\infty$ and $\varphi_{L}(\beta+\varepsilon)<0$ for some $\varepsilon \in(0, \alpha)$, then it holds for every $T \in(0, \infty]$ that

$$
\mathrm{P}\left(\sup _{0 \leq t \leq T} \int_{0}^{t} \mathrm{e}^{-L_{s}} \mathrm{~d} C_{s}>x\right) \sim \lambda \int_{0}^{T} \mathrm{P}\left(X \mathrm{e}^{-L_{t}}>x\right) \mathrm{d} t .
$$

Furthermore, $\sup _{0 \leq t \leq T} \int_{0}^{t} \mathrm{e}^{-L_{s}} \mathrm{~d} C_{s}$ follows a distribution in $\operatorname{ERV}(-\alpha,-\beta)$.

Proof. Let $\tau_{k}, k \in \mathbb{N}$, be the arrival times of the Poisson process $\left\{N_{t}, t \geq 0\right\}$. Then

$$
\sup _{0 \leq t \leq T} \int_{0}^{t} \mathrm{e}^{-L_{s}} \mathrm{~d} C_{s}=\sup _{0 \leq t \leq T} \sum_{k=1}^{N_{t}} X_{k} \mathrm{e}^{-L_{\tau_{k}}}=\max _{0 \leq n<\infty} \sum_{k=1}^{n} X_{k} \mathrm{e}^{-L_{\tau_{k}}} 1_{\left(\tau_{k} \leq T\right)},
$$

where, by convention, the summation over an empty set of indices produces a value 0 . This enables us to apply Lemma 3.4. By its convexity, $\varphi_{L}(u)<0$ for all $u \in(0, \beta+\varepsilon]$. Then it is straightforward to verify the corresponding conditions in order to apply Lemma 3.4. Therefore,

$$
\mathrm{P}\left(\sup _{0 \leq t \leq T} \int_{0}^{t} \mathrm{e}^{-L_{s}} \mathrm{~d} C_{s}>x\right) \sim \sum_{k=1}^{\infty} \mathrm{P}\left(X_{k} \mathrm{e}^{-L_{\tau_{k}}} 1_{\left(\tau_{k} \leq T\right)}>x\right)=\lambda \int_{0}^{T} \mathrm{P}\left(X \mathrm{e}^{-L_{t}}>x\right) \mathrm{d} t,
$$

where in the last step we used the fact that $\sum_{k=1}^{\infty} \mathrm{P}\left(\tau_{k} \in \mathrm{d} t\right)=\lambda \mathrm{d} t$.

The following lemma, which is a natural generalization of Lemma 2 of Grey (1994), does not require any information on the dependence structure of $(A, B)$ or on the left tail of $A$.

Lemma 3.6. Let $A, B$, and $Q$ be three random variables, with $Q$ independent of $(A, B)$. If each of $A$ and $Q$ follows a distribution in the class $\operatorname{ERV}(-\alpha,-\beta)$ for some $0<\alpha \leq \beta<\infty$, and $B$ is nonnegative, satisfying $\mathrm{E} B^{\beta+\varepsilon}<\infty$ for some $\varepsilon \in(0, \alpha)$, then the distribution of $A+Q B$ belongs to the class $\operatorname{ERV}(-\alpha,-\beta)$ and

$$
\mathrm{P}(A+Q B>x) \sim \mathrm{P}(A>x)+\mathrm{P}(Q B>x) .
$$


Proof. We only focus on the proof of relation (3.7) since the other conclusion follows immediately from relation (3.7) and the fact that the distribution of $Q B$ belongs to the class $\operatorname{ERV}(-\alpha,-\beta)$.

Arbitrarily choose an increasing function $l(\cdot):(0, \infty) \rightarrow(0, \infty)$ satisfying $l(x)<x / 2$, $l(x)=o(x)$, and $l(x) \rightarrow \infty$. Moreover, arbitrarily choose constants $\delta \in\left(0, \frac{1}{2}\right)$ and $p \in$ $(\beta /(\beta+\varepsilon), 1)$. We split the left-hand side of (3.7) as

$$
\begin{aligned}
\mathrm{P}(A+Q B>x)= & \mathrm{P}(A>(1+\delta) x)-\mathrm{P}(A>(1+\delta) x, A+Q B \leq x) \\
& +\mathrm{P}((1-\delta) x<A \leq(1+\delta) x, A+Q B>x) \\
& +\mathrm{P}(A<-l(x) \text { or } l(x)<A \leq(1-\delta) x, A+Q B>x) \\
& +\mathrm{P}(|A| \leq l(x), A+Q B>x) \\
= & I_{1}(x)-I_{2}(x)+I_{3}(x)+I_{4}(x)+I_{5}(x) .
\end{aligned}
$$

We estimate the five terms on the right-hand side of (3.8), in turn. Clearly,

$$
(1+\delta)^{-\beta} \mathrm{P}(A>x) \lesssim I_{1}(x) \lesssim(1+\delta)^{-\alpha} \mathrm{P}(A>x) .
$$

Noting that, by Lemma 3.1(ii), $x^{-p(\beta+\varepsilon)}=o(\mathrm{P}(A>x))$, we have

$$
\begin{aligned}
I_{2}(x) & =\mathrm{P}\left(A>(1+\delta) x, A+Q B \leq x,\left(B \leq x^{p}\right) \cup\left(B>x^{p}\right)\right) \\
& \leq \mathrm{P}(A>(1+\delta) x) \mathrm{P}\left(Q<-\delta x^{1-p}\right)+\mathrm{P}\left(B>x^{p}\right) \\
& =o(\mathrm{P}(A>(1+\delta) x))+O\left(x^{-p(\beta+\varepsilon)}\right) \\
& =o(\mathrm{P}(A>x)) .
\end{aligned}
$$

By the definition of the class $\operatorname{ERV}(-\alpha,-\beta)$,

$$
I_{3}(x) \leq \mathrm{P}((1-\delta) x<A \leq(1+\delta) x) \lesssim\left((1-\delta)^{-\beta}-(1+\delta)^{-\beta}\right) \mathrm{P}(A>x) .
$$

By conditioning on $A$ and applying Lemma 3.2, it holds for every $b>1$ and all large $x$ that

$$
\begin{aligned}
I_{4}(x) & \leq \mathrm{P}(|A|>l(x), Q B>\delta x) \\
& \leq b \mathrm{P}(Q>\delta x) \mathrm{E}\left(B^{\alpha-\varepsilon} \vee B^{\beta+\varepsilon}\right) 1_{(|A|>l(x))} \\
& =o(\mathrm{P}(Q>x)) .
\end{aligned}
$$

As in dealing with $I_{2}(x)$ above, we further split $I_{5}(x)$ into two parts according to $\left(B \leq x^{p}\right)$ and $\left(B>x^{p}\right)$. For the first part, we condition on $(A, B)$ and apply the uniformity over $|y| \leq l(x)$ of the asymptotic relation $\mathrm{P}(Q>x+y) \sim \mathrm{P}(Q>x)$. We have

$$
\begin{aligned}
I_{5}(x) & =\mathrm{P}\left(|A| \leq l(x), A+Q B>x,\left(B \leq x^{p}\right) \cup\left(B>x^{p}\right)\right) \\
& =(1+o(1)) \mathrm{P}\left(|A| \leq l(x), Q B>x, B \leq x^{p}\right)+O(1) \mathrm{P}\left(B>x^{p}\right) \\
& =(1+o(1)) \mathrm{P}(|A| \leq l(x), Q B>x)+o(1) \mathrm{P}(Q>x) \\
& =(1+o(1))(\mathrm{P}(Q B>x)-\mathrm{P}(|A|>l(x), Q B>x))+o(1) \mathrm{P}(Q>x) \\
& =(1+o(1)) \mathrm{P}(Q B>x)+o(1) \mathrm{P}(Q>x) \\
& \sim \mathrm{P}(Q B>x),
\end{aligned}
$$

where in the last but one step we used $\mathrm{P}(|A|>l(x), Q B>x)=o(1) \mathrm{P}(Q>x)$, as in the treatment of $I_{4}(x)$ above, and in the last step we used $\mathrm{P}(Q B>x) \asymp \mathrm{P}(Q>x)$. By simply substituting the estimates for $I_{1}(x), \ldots, I_{5}(x)$ into (3.8) and noting the arbitrariness of $\delta$, we obtain (3.7), as desired. 
The following lemma partially extends Theorem 1 of Grey (1994).

Lemma 3.7. Let $(A, B)$ be a random pair satisfying $\mathrm{E} \log (|A| \vee 1)<\infty, \mathrm{P}(B \geq 0)=1$, and $-\infty \leq \mathrm{E} \log B<0$. Let $Q$ be a random variable independent of $(A, B)$.

(i) Then there exists exactly one distribution for $Q$ satisfying the stochastic difference equation

$$
Q \stackrel{\mathrm{D}}{=} A+Q B
$$

where ' $\stackrel{\mathrm{D}}{=}$ 'denotes equality in distribution.

(ii) Furthermore, if the distribution of $A$ belongs to the class $\operatorname{ERV}(-\alpha,-\beta)$ for some $0<\alpha \leq \beta<\infty$ and $\mathrm{E}\left(B^{\alpha-\varepsilon} \vee B^{\beta+\varepsilon}\right)<1$ for some $\varepsilon \in(0, \alpha)$, then

$$
\mathrm{P}(Q>x) \sim \sum_{i=1}^{\infty} \mathrm{P}\left(A_{i} \prod_{j=1}^{i-1} B_{j}>x\right)
$$

where $\left\{\left(A_{k}, B_{k}\right), k \in \mathbb{N}\right\}$ is a sequence of i.i.d. copies of $(A, B)$ and, by convention, the multiplication over an empty set of indices produces a value 1.

Proof. (i) The existence and uniqueness of the weak solution of (3.9) are justified by Vervaat (1979, Theorem 1.6(b), (c) and Theorem 1.5(i)).

(ii) Let $\left\{Q_{k}, k \in \mathbb{N}\right\}$ be a sequence of random variables defined recursively by

$$
Q_{k}=A_{k}+Q_{k-1} B_{k}, \quad k \in \mathbb{N}
$$

where $Q_{0}$ is an arbitrary starting random variable independent of $\left\{\left(A_{k}, B_{k}\right), k \in \mathbb{N}\right\}$. Then by Vervaat (1979, Theorem $1.5(\mathrm{i})$ ), the sequence $\left\{Q_{k}, k \in \mathbb{N}\right\}$ weakly converges with a limit distribution which does not depend on $Q_{0}$ and coincides with the distribution of $Q$ in (3.9). See also Goldie (1991) for these statements. To prove relation (3.10), we apply the method developed by Grey (1994) to establish the following two relations:

$$
\mathrm{P}(Q>x) \lesssim \sum_{i=1}^{\infty} \mathrm{P}\left(A_{i} \prod_{j=1}^{i-1} B_{j}>x\right), \quad \mathrm{P}(Q>x) \gtrsim \sum_{i=1}^{\infty} \mathrm{P}\left(A_{i} \prod_{j=1}^{i-1} B_{j}>x\right)
$$

Let us prove the first relation in (3.12). Introduce a nonnegative random variable $Q_{0}^{\prime}$ independent of $(A, B)$ and satisfying $\mathrm{P}\left(Q_{0}^{\prime}>x\right) \sim c \mathrm{P}(A>x)$ for some constant $c>$ $\left(1-\mathrm{E}\left(B^{\alpha-\varepsilon} \vee B^{\beta+\varepsilon}\right)\right)^{-1}$. By Lemmas 3.2 and 3.6,

$$
\mathrm{P}\left(A+Q_{0}^{\prime} B>x\right) \lesssim\left(1+c \mathrm{E}\left(B^{\alpha-\varepsilon} \vee B^{\beta+\varepsilon}\right)\right) \mathrm{P}(A>x) .
$$

Since $1+c \mathrm{E}\left(B^{\alpha-\varepsilon} \vee B^{\beta+\varepsilon}\right)<c$, there exists some $x_{0}>0$ such that, for all $x>x_{0}$,

$$
\mathrm{P}\left(A+Q_{0}^{\prime} B>x\right) \leq \mathrm{P}\left(Q_{0}^{\prime}>x\right) .
$$

Construct a starting random variable $Q_{0}$ which is independent of $(A, B)$ and follows the distribution of $Q_{0}^{\prime}$ conditional on $Q_{0}^{\prime}>x_{0}$, which belongs to the class $\operatorname{ERV}(-\alpha,-\beta)$.

Substituting $Q_{0}$ into (3.11) and applying Lemma 3.6, the distribution of $Q_{1}$ belongs to the class $\operatorname{ERV}(-\alpha,-\beta)$ and

$$
\mathrm{P}\left(Q_{1}>x\right)=\mathrm{P}\left(A_{1}+Q_{0} B_{1}>x\right) \sim \mathrm{P}\left(A_{1}>x\right)+\mathrm{P}\left(Q_{0} B_{1}>x\right) .
$$


We claim that $Q_{1}$ is stochastically not greater than $Q_{0}$, written as $Q_{1} \leq_{\text {st }} Q_{0}$. Actually, for $x>x_{0}$,

$$
\begin{aligned}
\mathrm{P}\left(Q_{1}>x\right) & =\mathrm{P}\left(A+Q_{0}^{\prime} B>x \mid Q_{0}^{\prime}>x_{0}\right) \\
& \leq \frac{\mathrm{P}\left(A+Q_{0}^{\prime} B>x\right)}{\mathrm{P}\left(Q_{0}^{\prime}>x_{0}\right)} \\
& \leq \frac{\mathrm{P}\left(Q_{0}^{\prime}>x\right)}{\mathrm{P}\left(Q_{0}^{\prime}>x_{0}\right)} \\
& =\mathrm{P}\left(Q_{0}>x\right),
\end{aligned}
$$

while, for $x \leq x_{0}, \mathrm{P}\left(Q_{1}>x\right) \leq 1=\mathrm{P}\left(Q_{0}>x\right)$.

Using (3.11) with $k=2$ and $Q_{1} \leq_{\text {st }} Q_{0}$, we easily infer that $Q_{2} \leq_{\text {st }} Q_{1}$. Furthermore, by using Lemma 3.6 twice, the distribution of $Q_{2}$ belongs to the class $\operatorname{ERV}(-\alpha,-\beta)$ and

$$
\begin{aligned}
\mathrm{P}\left(Q_{2}>x\right) & \sim \mathrm{P}\left(A_{2}>x\right)+\mathrm{P}\left(Q_{1} B_{2}>x\right) \\
& =\mathrm{P}\left(A_{2}>x\right)+\mathrm{P}\left(A_{1} B_{2}+Q_{0} B_{1} B_{2}>x\right) \\
& \sim \mathrm{P}\left(A_{2}>x\right)+\mathrm{P}\left(A_{1} B_{2}>x\right)+\mathrm{P}\left(Q_{0} B_{1} B_{2}>x\right)
\end{aligned}
$$

Repeating this procedure, we can prove that the sequence $\left\{Q_{k}, k \in \mathbb{N}\right\}$, starting with $Q_{0}$, is stochastically nonincreasing and that, for each $k \in \mathbb{N}$, the distribution of $Q_{k}$ belongs to the class $\operatorname{ERV}(-\alpha,-\beta)$ with a tail satisfying

$$
\begin{aligned}
\mathrm{P}\left(Q_{k}>x\right) & \sim \sum_{i=1}^{k} \mathrm{P}\left(A_{i} \prod_{j=i+1}^{k} B_{j}>x\right)+\mathrm{P}\left(Q_{0} \prod_{j=1}^{k} B_{j}>x\right) \\
& =\sum_{i=1}^{k} \mathrm{P}\left(A_{i} \prod_{j=1}^{i-1} B_{j}>x\right)+\mathrm{P}\left(Q_{0} \prod_{j=1}^{k} B_{j}>x\right) .
\end{aligned}
$$

It follows that

$$
\mathrm{P}(Q>x) \lesssim \sum_{i=1}^{k} \mathrm{P}\left(A_{i} \prod_{j=1}^{i-1} B_{j}>x\right)+\mathrm{P}\left(Q_{0} \prod_{j=1}^{k} B_{j}>x\right) .
$$

The last tail probability is negligible as $k$ becomes large because, by Lemma 3.2,

$$
\mathrm{P}\left(Q_{0} \prod_{j=1}^{k} B_{j}>x\right) \lesssim c\left(\mathrm{E}\left(B^{\alpha-\varepsilon} \vee B^{\beta+\varepsilon}\right)\right)^{k} \mathrm{P}(A>x)
$$

and $\mathrm{E}\left(B^{\alpha-\varepsilon} \vee B^{\beta+\varepsilon}\right)<1$. This proves the first relation in (3.12).

We turn to the second relation in (3.12). The fact that $\mathrm{P}(Q>0)>0$ is explained in the proof of Theorem 1 of Grey (1994). Construct another starting random variable $Q_{0}$ independent of $(A, B)$ with tail

$$
\mathrm{P}\left(Q_{0}>x\right)=\mathrm{P}(Q>0) \mathrm{P}(A>x) 1_{(x \geq 0)}+\mathrm{P}(Q>x) 1_{(x<0)} .
$$

Clearly, the distribution of $Q_{0}$ belongs to the class $\operatorname{ERV}(-\alpha,-\beta)$ and $Q_{0} \leq_{\text {st }} Q$. It is easy to see that the sequence $\left\{Q_{k}, k \in \mathbb{N}\right\}$, starting with this $Q_{0}$, is stochastically bounded from above 
by $Q$. Similarly as before, applying Lemma 3.6 and relation (3.11) recursively, we obtain, for each $k \in \mathbb{N}$,

$$
\begin{aligned}
\mathrm{P}(Q>x) & \geq \mathrm{P}\left(Q_{k}>x\right) \\
& \sim \sum_{i=1}^{k} \mathrm{P}\left(A_{i} \prod_{j=1}^{i-1} B_{j}>x\right)+\mathrm{P}\left(Q_{0} \prod_{j=1}^{k} B_{j}>x\right) \\
& \geq \sum_{i=1}^{\infty} \mathrm{P}\left(A_{i} \prod_{j=1}^{i-1} B_{j}>x\right)-\sum_{i=k+1}^{\infty} \mathrm{P}\left(A_{i} \prod_{j=1}^{i-1} B_{j}>x\right) .
\end{aligned}
$$

By Lemma 3.2, the last sum above is negligible as $k$ becomes large. This proves the second relation in (3.12).

Finally, we list several useful martingale inequalities. The proof of the following lemma is an exercise of Doob's inequality; see also the proof of Lemma 3.2 of Paulsen (2002).

Lemma 3.8. Let $L$ be a Lévy process with Laplace exponent $\varphi_{L}(\cdot)$. If $\varphi_{L}(u)<\infty$ for some $u>0$ then $\mathrm{E}\left(\sup _{0 \leq t \leq T} \mathrm{e}^{-u L_{t}}\right)<\infty$ for every fixed $T \in(0, \infty)$.

For a stochastic process $M$, denote by $[M, M]$ and $\langle M, M\rangle$ its quadratic variation and predictable quadratic variation, respectively. The following lemma recalls some well-known martingale inequalities of which the first one is the Burkholder-Gundy inequality. Their proofs are given, for example, in Liptser and Shiryayev (1989).

Lemma 3.9. For a local martingale $M$, write $M_{T}^{*}=\sup _{0 \leq t \leq T}\left|M_{t}\right|$ for $0 \leq T \leq \infty$.

(i) For every $q \in(1, \infty)$, there exist positive constants $c_{q}$ and $c_{q}^{\prime}$ such that, uniformly for all local martingales $M$ with $M_{0}=0$ and all $0 \leq T \leq \infty$,

$$
c_{q}^{\prime} \mathrm{E}[M, M]_{T}^{q / 2} \leq \mathrm{E}\left(M_{T}^{*}\right)^{q} \leq c_{q} \mathrm{E}[M, M]_{T}^{q / 2} .
$$

Moreover, if $M_{t}$ is continuous then the inequalities above hold for all $0<q<\infty$.

(ii) If $M$ is a local square-integrable martingale with $M_{0}=0$, then it holds for every $q \in(0,2)$ that

$$
\mathrm{E}\left(M_{T}^{*}\right)^{q} \leq \frac{4-q}{2-q} \mathrm{E}\langle M, M\rangle_{T}^{q / 2} .
$$

\section{Proofs of the main results}

For the process $P$, the Lévy-Khintchine representation (2.1) for its characteristic exponent becomes

$$
\Psi_{P}(u)=\mathrm{i} p u+\frac{1}{2} \sigma_{P}^{2} u^{2}+\int_{|x|<1}\left(1-\mathrm{e}^{\mathrm{i} u x}+\mathrm{i} u x\right) v_{P}(\mathrm{~d} x)+\int_{|x| \geq 1}\left(1-\mathrm{e}^{\mathrm{i} u x}\right) v_{P}(\mathrm{~d} x) .
$$

Consequently, its Lévy-Itô decomposition is given by

$$
P_{t}=p t+\sigma_{P} W_{t}+M_{t}+C_{t},
$$

where $W$ is a standard Wiener process, $M$ is a square-integrable martingale with almost surely countably many jumps of magnitude less than 1 , and $C$ is a compound Poisson process of the form (3.6) in which the Poisson intensity is $\lambda^{*}=\nu_{P}(\mathbb{R} \backslash(-1,1))$ and $F$ is given by $v_{P}(\cdot) 1_{\mathbb{R} \backslash(-1,1)} / \lambda^{*}$. In particular, $W, M$, and $C$ are three independent Lévy processes. See, e.g. Kyprianou (2006) for more details. 


\subsection{Proof of Theorem $2.1(i)$}

Recall relation (1.3) with $T<\infty$. The basic idea of our proof is that, when considering the tail behavior of $Z_{T}^{*}$, the Wiener process and small jumps of the process $P$ are negligible.

Clearly, by (4.1), it holds that

$$
\sum_{j=1}^{3} \inf _{0 \leq t \leq T} I_{j, t}+\sup _{0 \leq t \leq T} I_{4, t} \leq Z_{T}^{*} \leq \sum_{j=1}^{4} \sup _{0 \leq t \leq T} I_{j, t},
$$

where

and

$$
\begin{aligned}
& I_{1, t}=p \int_{0}^{t} \mathrm{e}^{-\tilde{R}_{s}} \mathrm{~d} s, \\
& I_{2, t}=\sigma_{P} \int_{0}^{t} \mathrm{e}^{-\tilde{R}_{s}} \mathrm{~d} W_{s}, \\
& I_{3, t}=\int_{0}^{t} \mathrm{e}^{-\tilde{R}_{s}} \mathrm{~d} M_{s},
\end{aligned}
$$

$$
I_{4, t}=\int_{0}^{t} \mathrm{e}^{-\tilde{R}_{s}} \mathrm{~d} C_{s}
$$

By Lemma 3.5, the distribution of $\sup _{0 \leq t \leq T} I_{4, t}$ belongs to the class $\operatorname{ERV}(-\alpha,-\beta)$ and

$$
\mathrm{P}\left(\sup _{0 \leq t \leq T} I_{4, t}>x\right) \sim \lambda^{*} \int_{0}^{T} \mathrm{P}\left(X^{*} \mathrm{e}^{-\tilde{R}_{t}}>x\right) \mathrm{d} t,
$$

where $X^{*}$, independent of $\tilde{R}$, follows the distribution $F$ as defined above. Note that the righthand side of (4.3) with $x>0$ is identical to the right-hand side of (2.3). If

$$
\mathrm{E}\left(\sup _{0 \leq t \leq T}\left|I_{j, t}\right|^{\beta+\varepsilon}\right)<\infty, \quad j=1,2,3,
$$

then, by Lemma 3.1(ii) and Lemma 3.3, all terms except $\sup _{0 \leq t \leq T} I_{4, t}$ appearing in the upper and lower bounds for $Z_{T}^{*}$ in (4.2) are negligible and it follows from (4.3) that

$$
\mathrm{P}\left(Z_{T}^{*}>x\right) \sim \mathrm{P}\left(\sup _{0 \leq t \leq T} I_{4, t}>x\right) \sim \lambda \int_{0}^{T} \mathrm{P}\left(X \mathrm{e}^{-\tilde{R}_{t}}>x\right) \mathrm{d} t,
$$

yielding relation (2.3). Therefore, it suffices to prove (4.4).

By Lemma 3.8 we have

$$
\mathrm{E}\left(\sup _{0 \leq t \leq T} \mathrm{e}^{-(\beta+\varepsilon) \tilde{R}_{t}}\right)<\infty .
$$

Then relation (4.4) with $j=1$ follows trivially from (4.5). Since $I_{2, t}$ is a continuous martingale and $\left[I_{2, t}, I_{2, t}\right]=\sigma_{P}^{2} \int_{0}^{t} \mathrm{e}^{-2 \tilde{R}_{s}} \mathrm{~d} s$, we use Lemma 3.9(i) and relation (4.5) again to obtain, for some constant $c_{1}>0$,

$$
\begin{aligned}
\mathrm{E}\left(\sup _{0 \leq t \leq T}\left|I_{2, t}\right|^{\beta+\varepsilon}\right) & \leq c_{1} \mathrm{E}\left(\int_{0}^{T} \mathrm{e}^{-2 \tilde{R}_{t}} \mathrm{~d} t\right)^{(\beta+\varepsilon) / 2} \\
& \leq c_{1} T^{(\beta+\varepsilon) / 2} \mathrm{E}\left(\sup _{0 \leq t \leq T} \mathrm{e}^{-(\beta+\varepsilon) \tilde{R}_{t}}\right) \\
& <\infty
\end{aligned}
$$


Similarly, by Lemma 3.9 and relation (4.5), it holds for some constant $c_{2}>0$ that

$$
\mathrm{E}\left(\sup _{0 \leq t \leq T}\left|I_{3, t}\right|^{\beta+\varepsilon}\right) \leq c_{2}\left(\int_{|x| \leq 1} x^{2} v_{P}(\mathrm{~d} x)\right)^{(\beta+\varepsilon) / 2} \mathrm{E}\left(\sup _{0 \leq t \leq T} \mathrm{e}^{-(\beta+\varepsilon) \tilde{R}_{t}}\right)<\infty .
$$

\subsection{Proof of Theorem 2.1(ii)}

Recall (1.3) with $T=\infty$, that is, $\psi(x, \infty)=\mathrm{P}\left(Z_{\infty}^{*}>x\right)$. The basic idea of our proof is to construct two discrete-time processes, fulfilling a certain recursive structure, whose limits serve as the stochastic upper and lower bounds, respectively, for the ultimate supremum of the discounted net loss process. This idea is from Grey (1994).

To derive an asymptotic upper bound for $Z_{\infty}^{*}$, we observe that

$$
Z_{\infty}^{*} \leq Z_{1}^{*}+\mathrm{e}^{-\tilde{R}_{1}} \sup _{1 \leq t<\infty} \int_{1}^{t} \mathrm{e}^{-\left(\tilde{R}_{s}-\tilde{R}_{1}\right)} \mathrm{d} P_{s} \stackrel{\mathrm{D}}{=} Z_{1}^{*}+Z_{\infty}^{*} \mathrm{e}^{-\tilde{R}_{1}}
$$

where on the right-hand side $Z_{\infty}^{*}$ is independent of $\left(Z_{1}^{*}, \tilde{R}_{1}\right)$. Consider the stochastic difference equation

$$
Q^{*} \stackrel{\mathrm{D}}{=} Z_{1}^{*}+Q^{*} \mathrm{e}^{-\tilde{R}_{1}},
$$

where on the right-hand side $Q^{*}$ is independent of $\left(Z_{1}^{*}, \tilde{R}_{1}\right)$. By Theorem 2.1(i), the distribution of $Z_{1}^{*}$ belongs to the class $\operatorname{ERV}(-\alpha,-\beta)$ and

$$
\mathrm{P}\left(Z_{1}^{*}>x\right) \sim \lambda \int_{0}^{1} \mathrm{P}\left(X \mathrm{e}^{-\tilde{R}_{t}}>x\right) \mathrm{d} t .
$$

By comparing (4.6) with (4.7) and applying Lemma 3.7, we have

$$
\mathrm{P}\left(Z_{\infty}^{*}>x\right) \leq \mathrm{P}\left(Q^{*}>x\right) \sim \sum_{i=1}^{\infty} \mathrm{P}\left(A_{i} \prod_{j=1}^{i-1} B_{j}>x\right),
$$

where $\left\{\left(A_{k}, B_{k}\right), k \in \mathbb{N}\right\}$ is a sequence of i.i.d. copies of the random pair $\left(Z_{1}^{*}, \mathrm{e}^{-\tilde{R}_{1}}\right)$. It follows from (4.8) and (4.9) that, for arbitrarily fixed $\delta>0$ and some large $x_{0}$,

$$
\begin{aligned}
\mathrm{P}\left(Z_{\infty}^{*}>x\right) \lesssim & \sum_{i=1}^{\infty} \mathrm{P}\left(A_{i} \prod_{j=1}^{i-1} B_{j}>x, \prod_{j=1}^{i-1} B_{j} \leq \frac{x}{x_{0}}\right)+\sum_{i=1}^{\infty} \mathrm{P}\left(\prod_{j=1}^{i-1} B_{j}>\frac{x}{x_{0}}\right) \\
\leq & (1+\delta) \lambda \sum_{i=1}^{\infty} \int_{i-1}^{i} \mathrm{P}\left(X \mathrm{e}^{-\left(\tilde{R}_{t}-\tilde{R}_{i-1}\right)} \prod_{j=1}^{i-1} B_{j}>x\right) \mathrm{d} t \\
& +\left(\frac{x}{x_{0}}\right)^{-(\beta+\varepsilon)} \sum_{i=1}^{\infty} \mathrm{E}\left(\prod_{j=1}^{i-1} B_{j}^{\beta+\varepsilon}\right) \\
= & (1+\delta) \lambda \int_{0}^{\infty} \mathrm{P}\left(X \mathrm{e}^{-\tilde{R}_{t}}>x\right) \mathrm{d} t+\frac{\left(x / x_{0}\right)^{-(\beta+\varepsilon)}}{1-\mathrm{E}^{-(\beta+\varepsilon) \tilde{R}_{1}}} .
\end{aligned}
$$

Since the last term above is negligible and $\delta$ can be arbitrarily small, we obtain

$$
\psi(x, \infty) \lesssim \lambda \int_{0}^{\infty} \mathrm{P}\left(X \mathrm{e}^{-\tilde{R}_{t}}>x\right) \mathrm{d} t
$$


To derive an asymptotic lower bound, by Theorem 2.1(i) we have, for every $T>0$,

$$
\mathrm{P}\left(Z_{\infty}^{*}>x\right) \geq \mathrm{P}\left(Z_{T}^{*}>x\right) \sim \lambda\left(\int_{0}^{\infty}-\int_{T}^{\infty}\right) \mathrm{P}\left(X \mathrm{e}^{-\tilde{R}_{t}}>x\right) \mathrm{d} t .
$$

By Lemma 3.2, it holds for every $b>1$ and all large $x$ that

$$
\begin{aligned}
\int_{T}^{\infty} \frac{\mathrm{P}\left(X \mathrm{e}^{-\tilde{R}_{t}}>x\right)}{\mathrm{P}(X>x)} \mathrm{d} t & \leq b \int_{T}^{\infty} \mathrm{E}\left(\mathrm{e}^{-(\alpha-\varepsilon) \tilde{R}_{t}} \vee \mathrm{e}^{-(\beta+\varepsilon) \tilde{R}_{t}}\right) \mathrm{d} t \\
& \leq b \int_{T}^{\infty}\left(\mathrm{e}^{\varphi_{\tilde{R}}(\alpha-\varepsilon) t}+\mathrm{e}^{\varphi_{\tilde{R}}(\beta+\varepsilon) t}\right) \mathrm{d} t \\
& =b\left(\frac{\mathrm{e}^{\varphi_{\tilde{R}}(\alpha-\varepsilon) T}}{-\varphi_{\tilde{R}}(\alpha-\varepsilon)}+\frac{\mathrm{e}^{\varphi_{\tilde{R}}(\beta+\varepsilon) T}}{-\varphi_{\tilde{R}}(\beta+\varepsilon)}\right)
\end{aligned}
$$

since $\varphi_{\tilde{R}}(\alpha-\varepsilon)<0$ and $\varphi_{\tilde{R}}(\beta+\varepsilon)<0$. This means that, as $T$ becomes large, the second term on the right-hand side of (4.10) is negligible when compared with $\mathrm{P}(X>x)$ and, hence, with $\int_{0}^{\infty} \mathrm{P}\left(X \mathrm{e}^{-\tilde{R}_{t}}>x\right) \mathrm{d} t$. It follows that

$$
\psi(x, \infty) \gtrsim \lambda \int_{0}^{\infty} \mathrm{P}\left(X \mathrm{e}^{-\tilde{R}_{t}}>x\right) \mathrm{d} t .
$$

\subsection{Proof of Corollary 2.1}

We start with (2.3), which holds for $T \in(0, \infty)$. By Lemma 3.2, it holds for every $b>1$ and all large $x$ that

$$
\frac{\mathrm{P}\left(X \mathrm{e}^{-\tilde{R}_{t}}>x\right)}{\mathrm{P}(X>x)} \leq b \mathrm{E}\left(\mathrm{e}^{-(\alpha-\varepsilon) \tilde{R}_{t}} \vee \mathrm{e}^{-(\alpha+\varepsilon) \tilde{R}_{t}}\right) \leq b\left(\mathrm{e}^{\varphi_{\tilde{R}}^{(\alpha-\varepsilon) t}}+\mathrm{e}^{\varphi_{\tilde{R}}(\beta+\varepsilon) t}\right),
$$

the right-hand side of which is integrable with respect to $\mathrm{d} t$ over $[0, \infty)$. Therefore, by the dominated convergence theorem,

$$
\lim _{x \rightarrow \infty} \frac{\psi(x, T)}{\mathrm{P}(X>x)}=\lambda \int_{0}^{T} \lim _{x \rightarrow \infty} \frac{\mathrm{P}\left(X \mathrm{e}^{-\tilde{R}_{t}}>x\right)}{\mathrm{P}(X>x)} \mathrm{d} t=\lambda \int_{0}^{T} \mathrm{E}^{-\alpha \tilde{R}_{t}} \mathrm{~d} t=\lambda \frac{1-\mathrm{e}^{\varphi_{\tilde{R}}(\alpha) T}}{-\varphi_{\tilde{R}}(\alpha)},
$$

where the second step is due to the well-known Breiman's theorem (see Breiman (1965)).

\section{Acknowledgements}

The authors would like to thank the Editor and an anonymous referee for their careful reading and useful comments. Hao acknowledges the support of the Natural Sciences and Engineering Research Council of Canada (grant number 386552-2010) and Tang acknowledges the support by a Centers of Actuarial Excellence (CAE) research grant (2013-2015) from the Society of Actuaries.

\section{References}

Albrecher, H., Constantinescu, C. and Thomann, E. (2012). Asymptotic results for renewal risk models with risky investments. Stoch. Process. Appl. 122, 3767-3789.

Asmussen, S. (1998). Subexponential asymptotics for stochastic processes: extremal behavior, stationary distributions and first passage probabilities. Ann. Appl. Prob. 8, 354-374. 
Asmussen, S. And Albrecher, H. (2010). Ruin Probabilities, 2nd edn. World Scientific, Hackensack, NJ.

Bingham, N. H., Goldie, C. M. And Teugels, J. L. (1987). Regular Variation. Cambridge University Press.

Breiman, L. (1965). On some limit theorems similar to the arc-sin law. Theory Prob. Appl. 10, 323-331.

CHEN, Y. AND YUEN, K. C. (2009). Sums of pairwise quasi-asymptotically independent random variables with consistent variation. Stoch. Models 25, 76-89.

Cline, D. B. H. And Samorodnitsky, G. (1994). Subexponentiality of the product of independent random variables. Stoch. Process. Appl. 49, 75-98.

Frolova, A., Kabanov, Y. and Pergamenshchikov, S. (2002). In the insurance business risky investments are dangerous. Finance Stoch. 6, 227-235.

Goldie, C. M. (1991). Implicit renewal theory and tails of solutions of random equations. Ann. Appl. Prob. 1, $126-166$.

Grey, D. R. (1994). Regular variation in the tail behaviour of solutions of random difference equations. Ann. Appl. Prob. 4, 169-183.

Heyde, C. C. AND WANG, D. (2009). Finite-time ruin probability with an exponential Lévy process investment return and heavy-tailed claims. Adv. Appl. Prob. 41, 206-224.

Hult, H. AND LindsKog, F. (2011). Ruin probabilities under general investments and heavy-tailed claims. Finance Stoch. 15, 243-265.

Kalashnikov, V. and Konstantinides, D. (2000). Ruin under interest force and subexponential claims: a simple treatment. Insurance Math. Econom. 27, 145-149.

Kalashnikov, V. AND Norberg, R. (2002). Power tailed ruin probabilities in the presence of risky investments. Stoch. Process. Appl. 98, 211-228.

KlÜPPElberg, C. AND Kostadinova, R. (2008). Integrated insurance risk models with exponential Lévy investment. Insurance Math. Econom. 42, 560-577.

KlÜPPELbERG, C. AND STADTMÜLleR, U. (1998). Ruin probabilities in the presence of heavy-tails and interest rates. Scand. Actuarial J. 1998, 49-58.

Konstantinides, D., Tang, Q. And Tsitsiashvili, G. (2002). Estimates for the ruin probability in the classical risk model with constant interest force in the presence of heavy tails. Insurance Math. Econom. 31, 447-460.

Kyprianou, A. E. (2006). Introductory Lectures on Fluctuations of Lévy Processes with Applications. Springer, Berlin.

LiPTSER, R. S. AND ShIRYAYev, A. N. (1989). Theory of Martingales. Kluwer, Dordrecht.

Olvera-Cravioto, M. (2012). Asymptotics for weighted random sums. Adv. Appl. Prob. 44, 1142-1172.

PAulsen, J. (1998a). Ruin theory with compounding assets-a survey. The interplay between insurance, finance and control. Insurance Math. Econom. 22, 3-16.

PAulsen, J. (1998b). Sharp conditions for certain ruin in a risk process with stochastic return on investments. Stoch. Process. Appl. 75, 135-148.

PAulsen, J. (2002). On Cramér-like asymptotics for risk processes with stochastic return on investments. Ann. Appl. Prob. 12, 1247-1260.

Paulsen, J. (2008). Ruin models with investment income. Prob. Surveys 5, 416-434.

Protter, P. E. (2005). Stochastic Integration and Differential Equations, 2nd edn. Springer, Berlin.

RESNICK, S. I. AND WiLleKens, E. (1991). Moving averages with random coefficients and random coefficient autoregressive models. Commun. Statist. Stoch. Models 7, 511-525.

Samorodnitsky, G. And Taqqu, M. S. (1994). Stable Non-Gaussian Random Processes. Chapman \& Hall, New York.

TANG, Q. (2005). The finite-time ruin probability of the compound Poisson model with constant interest force. J. Appl. Prob. 42, 608-619.

TANG, Q. AND Tsitsiashvili, G. (2003). Precise estimates for the ruin probability in finite horizon in a discrete-time model with heavy-tailed insurance and financial risks. Stoch. Process. Appl. 108, 299-325.

TANG, Q., Wang, G. And Yuen, K. C. (2010). Uniform tail asymptotics for the stochastic present value of aggregate claims in the renewal risk model. Insurance Math. Econom. 46, 362-370.

VERVAAT, W. (1979). On a stochastic difference equation and a representation of nonnegative infinitely divisible random variables. Adv. Appl. Prob. 11, 750-783.

Zhang, Y., Shen, X. AND Weng, C. (2009). Approximation of the tail probability of randomly weighted sums and applications. Stoch. Process. Appl. 119, 655-675. 